\title{
Mit Blick auf die Geste - multimodale Verfestigungen in der Interaktion
}

\section{Einleitung}

In der Interaktionalen Linguistik herrscht ein breiter Konsens, dass Grammatik und Interaktion in einem sich wechselseitig bedingenden Verhältnis stehen (vgl. Auer i. d. B.; Couper-Kuhlen/Selting 2001; Couper-Kuhlen/Thompson i. d. B.; Deppermann i. d. B.; Deppermann/Fiehler/Spranz-Fogasy 2006; Günthner/Imo 2006; Selting/Couper-Kuhlen 2000). Grammatische Strukturen sind auf die Interaktion zugeschnitten und in historischen Sedimentierungsprozessen aus ihr hervorgegangen. Folglich weisen Grammatik und Interaktion fundamentale strukturelle Homologien auf (Auer 2005: 7). Aus Sicht der Konversationsanalyse, der Ethnomethodologie und der Interaktionalen Linguistik bilden neben der gesprochenen Sprache körperlich-visuelle Ausdrucksressourcen wie Gestik, Mimik, Blickverhalten, Körperbewegungen, Objektmanipulation etc. einen konstitutiven Bestandteil sozialer Interaktion. Ungeklärt ist allerdings die Frage, inwieweit multimodale Phänomene zur Grammatikbeschreibung dazugehören. Während die Prosodie in konstruktionsgrammatischen Darstellungen zum Bestandteil von grammatischen Einheiten bzw. Konstruktionen gerechnet wird (Auer 2006), ist die Integration leiblicher Ausdrucksressourcen und Praktiken, von Räumlichkeit und Materialität in die Grammatik umstritten. Konstruktionsgrammatische Bemühungen um eine multimodal erweiterte Auffassung von Konstruktionen (vgl. Zima 2014, Zima 2017; Zima/Bergs 2017) erweisen sich nur dann als überzeugend, wenn verbale und leibliche Komponenten über statistische Kookkurrenz-Häufigkeiten hinaus als „'non-accidental'“ (Ningelgen/Auer 2017: 1) gelten können, einen essentiellen Beitrag zur Konstruktion leisten und Teil des linguistischen Wissens sind.

Welche in der Interaktion rekurrent begegnenden multimodalen Gestalten tatsächlich eine grammatische Einheit bilden oder unterwegs sind zur Grammatikalisierung (Auer i.d. B.; Hopper/Traugott 1993), ist daher anhand empirischer Untersuchungen erst noch nachzuweisen und keineswegs für das gesamte Inventar verbaler und kookkurrenter leiblicher Praktiken zu postulieren, selbst wenn ein ausgesprochen weiter Grammatikbegriff zugrunde gelegt würde. Hingegen zeigen Untersuchungen zum primordialen Gebrauch demonstrativer, lokal- und modaldeiktischer Ausdrücke in der face-to-face-Interaktion (Stukenbrock 2009a, b, 2010, 2014, 2015), dass es Klassen von linguistischen Elementen gibt, die qua 
Zugehörigkeit zum Zeigfeld der Sprache (Bühler 1965 [1934]) in ihrem Entstehungs- und Grammatikalisierungsprozess multimodal konstituiert sind und bei denen verbale und leibliche Komponenten einen essentiellen Bestandteil der Konstruktion bilden.

Bereits Goodwin (1996) argumentiert in seinem Aufsatz „Transparent Vision“ für ein über die Satzgrammatik hinausgehendes Verständnis von Grammatik und schlägt das Konzept einer „interactionally situated grammar“ vor: „[T] he scope of the term grammar will not be limited to phenomena within the stream of speech, but will also encompass structures providing for the organization of endogenous activity systems within which strips of talk are embedded" (Goodwin 1996: 370). Am Beispiel sogenannter ,prospective indexicals“ (Goodwin 1996: 384), einer Gruppe grammatisch heterogener Ausdrücke, die von Nominalphrasen über deiktische Ausdrücke bis zu response cries (Goffman 1981) reichen, weist er nach, dass deren Bedeutung erst prospektiv und reflexiv im emergent von den Beteiligten konstituierten lokalen Aktivitätskontext interaktiv hervorgebracht wird. Problematisch bleibt dabei jedoch der unklare Grammatikbegriff, dessen Ausweitung über die Satzgrenzen hinaus nicht dahingehend bestimmt wird, welche Bestandteile des interaktiven Feldes mit zu integrieren und wo die Grenzen zu ziehen seien.

Auch jüngste konversationsanalytische Versuche, den Grammatikbegriff dergestalt auszuweiten, dass Körperbewegungen (Keevallik 2018) in die grammatische Strukturbeschreibung mit eingehen sollen, stehen vor dem Dilemma potenzieller Grenzenlosigkeit und der Gefahr eines schließlich ins Metaphorische ausgedehnten Grammatikbegriffs (Couper-Kuhlen 2018: 24). In ihrer Replik auf Keevallik argumentiert Couper-Kuhlen, dass positionelle Sensibilität von Grammatik (,positional sensitivity of grammar“; vgl. auch Schegloff 1996) zwar leibliches Verhalten miteinschließen und dass Letzteres zweifelsohne in grammatisch projizierten Slots auftreten könne. Dies führe aber nicht zu dem Schluss, leibliche Praktiken seien selbst grammatisch: „Grammar must be able to account for the slot but not necessarily for what fills it" (Couper-Kuhlen 2018: 24). Entsprechend unterscheidet Auer zwischen Projektionen in der Grammatik und Projektionen in der Interaktion: Während Erstere auf grammatischem Wissen über formale Organisationsprinzipien und daraus abgeleiteten Fortsetzungserwartungen basieren, gründen Letztere im Wissen der Beteiligten über die Sequenzierung von Aktivitäten (Auer 2005: 27). Grammatische Strukturen können Projektionen aufbauen, die durch leibliche Praktiken eingelöst werden, der umgekehrte Fall hingegen, dass spezifische grammatische Strukturen durch sequenziell vorangehende leibliche Aktivitäten projiziert werden, erweist sich interaktionslinguistisch als problematisch. 
Hinzu kommt das Problem, dass sich Beobachtungen zum reflexiven Konstitutionszusammenhang von konkreten grammatischen Ressourcen und lokal emergierenden leiblichen, räumlichen und temporalen Interaktionskontexten nicht ohne Weiteres auf die umfangreichen Erkenntnisse der Grammatikalisierungsforschung beziehen lassen. Letztere hat die Emergenz grammatischer Elemente und Strukturen aus lexikalischen Einheiten im Diskurs beschrieben und Grammatikalisierungsparameter zum Nachweis (verschiedener Stadien) von Grammatikalisierungsprozessen definiert (Diewald 1997; Lehmann 1995; Hopper/ Traugott 1993), die sich in ganz anderen zeitlichen Dimensionen, oft über Jahrhunderte hinweg, vollziehen. So argumentiert Streeck (2018) in Auseinandersetzung mit Keevallik (2018), dass der Nachweis einer Entstehung von Grammatik aus leiblichen Handlungen und Aktivitätskontexten zum einen kontextspezifische Einzeldarstellungen übersteigen und kontextunabhängigere Beschreibungen leisten müsse. Zum anderen erfordere er historische Analysen zur Emergenz grammatischer Strukturen über größere Zeitspannen hinweg.

Die folgende Untersuchung leistet einen Beitrag zu dieser Diskussion, indem sie multimodale Verfestigungen analysiert, die sich im Verlauf der Interaktionsgeschichte zwischen einer Trainerin und ihren Schülerinnen im Kontext schulischer Selbstverteidigungstrainings sedimentieren. Es soll gezeigt werden, dass diese in der lokalen Interaktion für die Beteiligten zu gestalthaften Einheiten werden, die spezifische Projektionen aufbauen. Im Gegensatz zu Keevallik (2018) wird für diese aus einer progressiven Routinisierung hervorgehenden Sedimentierungen jedoch nicht der Status grammatikalisierter Einheiten bzw. Strukturen reklamiert, sondern an der Unterscheidung zwischen Grammatikalisierung als Herausbildung kontextunabhängiger Formen einerseits und aus Routinisierung und Sedimentierung hervorgehenden Formaten anderseits festgehalten. Letztere mögen unter Umständen einen Grammatikalisierungprozess durchlaufen, bei dem neben der formalen Reduktion weitere Veränderungen auf dem Kontinuum der Grammatikalisierungsparameter betroffen sind, oder sie verschwinden wieder.

Nach einem kurzen Abschnitt zu Multimodalität in Instruktionen widmet sich die empirische Analyse erstens einer multimodalen Konstruktion, deren verbale Komponente das Modaldeiktikon so bildet und die in den Instruktionen der Trainerin eine zentrale interpersonelle Koordinierungsfunktion ausübt. Zweitens werden die sich im mikrointeraktionsgeschichtlichen Verlauf herausbildenden Routinisierungen, temporalen Verdichtungen und formalen Reduktionen im Instruktionsformat betrachtet, die als Ergebnis eines lokalen Sedimentierungsprozesses aufgefasst werden. 


\section{Multimodalität in Instruktionen}

Als grundlegende Eigenschaft von face-to-face-Interaktion spielt die Multimodalität in mündlichen Instruktionen kopräsenter Interaktionspartner eine herausgehobene Rolle. Eine Person in etwas zu unterweisen, sie zu instruieren, was sie tun soll, was sie wann und wie tun soll (Stukenbrock 2014), ist eine Aktivität, die grundlegend auf eine interaktionsräumliche Konfiguration angewiesen ist, die Goffman „an eye-to-eye ecological huddle“ (Goffman 1963: 95) genannt hat. Sie gewährleistet eine visuelle Orientierung der Interaktionsbeteiligten aufeinander, ermöglicht ihnen fortwährende wechselseitige Wahrnehmung („mutual monitoring“; vgl. M. Goodwin 1980) sowie Momente der „Wahrnehmungswahrnehmung“ (Luhmann 1984; Hausendorf 2003; Stukenbrock 2009a, 2015). Wechselseitige Wahrnehmung ist für die Beteiligten besonders in Instruktionssequenzen relevant, deren Vermittlungsgegenstand körperliche Praktiken (Bewegungsabläufe, instrumentelle Handlungen, Handhabung von Objekten etc.) darstellen. Leibliche Ausdrucksressourcen werden in Verbindung mit Sprache als integraler Bestandteil multimodaler Äußerungskonstruktion und Handlungskonstitution mobilisiert. Zugleich konstituieren Körper, Körperbewegungen, körperliche Verhaltensweisen, deren Möglichkeiten und Grenzen thematisch den Gegenstand von Kommunikation, Demonstration und Evaluation. Leibliches ist sowohl Medium als auch Gegenstand multimodaler Instruktionssequenzen, wobei der Leib von instruierender und instruierter Person in ein spezifisches Verhältnis der Zwischenleiblichkeit treten (Meyer/Streeck/Jordan 2017).

In der ethnomethodologischen und konversationsanalytischen Forschungsliteratur werden Instruktionen sequenziell als Paarsequenzen beschrieben. Demnach fordert der erste Paarteil, von Garfinkel (2002 [1967]) „instruction“, von Goodwin (2006) und Mondada (2011) als „directive“ bezeichnet, als zweiten Paarteil die von Goodwin „response“, Garfinkel ,instructed“ bzw. „,following“ und von Mondada „complying action“ genannte Folgehandlung. Videoanalytische Untersuchungen zu multimodalen Instruktionssequenzen zeigen, dass die konditionell relevant gesetzten zweiten Paarteile oftmals nicht als sprachliche Äußerungen, sondern leiblich realisiert werden (Lindwall/Ekström 2012; Mondada 2009, 2011; De Stefani/Gazin 2014; Rauniomaa/Keisanen 2012; Stukenbrock 2014).

Gegenstand der folgenden Untersuchung sind mündliche Instruktionssequenzen, die im institutionellen Rahmen schulischer Interaktion vollzogen werden. Erklären, Zeigen und Demonstrieren (Stukenbrock 2009b) sind die dominanten, institutionell verankerten Aktivitäten, die an bestimmte Rollen, Beteiligungsformate und sequenzielle Strukturen gebunden sind. 
Datengrundlage bilden 12 Stunden Videoaufnahmen von Selbstverteidigungskursen für 12- bis 16-jährige Mädchen, die an verschiedenen deutschen Schulen durchgeführt wurden und die Vermittlung körperlicher Techniken und Fähigkeiten zur Abwehr eines Aggressors in bedrohlichen Situationen (Bushaltestelle, nächtlicher Heimweg etc.) zum Gegenstand haben. Die Kursstunden vollziehen sich in aufeinander aufbauenden Phasen (Demonstrationsphasen, Präparationsphasen, Übungsphasen, Erläuterungsphasen, Revisionsphasen, Reflexionsphasen), in denen unterschiedliche Beteiligungsformate auftreten. Solodemonstrationen der Trainerin werden ergänzt durch Partnerdemonstrationen, in denen eine Assistentin eine komplementäre Rolle übernimmt und unter der Regie der Trainerin mit ihr gemeinsam bestimmte Bewegungsabläufe vorführt. Die Schülerinnen werden in Solo-, Partner- und chorisch organisierten Übungen Schritt für Schritt an die zu lernenden Bewegungsmuster herangeführt. Dabei variieren Typus, Komplexität und Reichweite, sprachliche und körperliche Realisierungsformate der Instruktionen. Während in frühen Phasen zunächst kleinteilig Einzelbewegungen demonstriert und geübt werden, treten in späteren Phasen zunehmend komplexere Bewegungsformate und -abläufe hinzu, die sich aus den zuvor geübten Einzelpraktiken zusammensetzen. Damit gehen unterschiedliche räumliche Arrangements der Gruppen und Untergruppen in der Turnhalle einher.

Multimodale Instruktionen der Trainerin und instruierte Folgehandlungen der Mädchen treten in zwei unterschiedlichen Sequenzformaten auf. Den ersten Typ bilden instruierende Handlungen, in denen die Trainerin verbale Erklärungen und leibliche Demonstrationen miteinander kombiniert und zu dem Zweck enaktiert, dass sie von den Mädchen zunächst nur wahrgenommen und als Vorausschau auf künftige Übungssequenzen wahrgenommen, aber nicht unmittelbar nachgemacht werden. Diese kontrastieren mit einem zweiten Sequenztyp, in dem von Erklärungen begleitete leibliche Demonstrationen als instruierender erster Paarteil gestaltet werden, der als zweiten Paarteil eine von den Schülerinnen kollektiv auszuführende instruierte Handlung konditionell relevant macht, die unmittelbar auf den ersten Paarteil zu erfolgen hat. Die im Folgenden präsentierten Analysen beziehen sich ausschließlich auf den zweiten Sequenztyp, bei dem sich an die multimodale Instruktion aus leiblicher Demonstration und begleitender Verbalinstruktion direkt die gemeinsame, chorische Ausführung der Schülerinnen anschließt. Es wird gezeigt, dass die instruierende Handlung multimodal konstituiert ist und als solche im interaktionsgeschichtlichen Verlauf formal reduziert und temporal verdichtet wird. Dies verändert auch die temporale Beziehung zur instruierten Handlung, die ihrerseits durch mehrfache Wiederholungen quasi automatisiert wird, mit dem Ergebnis, dass schließlich die Instruktionssequenz als Ganze, d.h. die Abfolge zwischen erstem und zweitem Paarteil zwischen wiederholten Durchgängen, eine Routinisierung erfährt. 


\section{Analysen: Multimodale Instruktionen leiblicher Praktiken}

\subsection{Praktiken der Vermittlung zwischen eigenem und fremdem Leib - zwischenleibliche Vermittlungspraktiken}

Eine Besonderheit der analysierten Instruktionssequenzen besteht darin, dass die Instruierten nicht nur bestimmte Selbstverteidigungspraktiken lernen, die in den jeweiligen Sequenzen den konkreten Vermittlungsgegenstand des übergreifenden Lernziels erfolgreicher Selbstverteidigung bilden. Die These lautet, dass sie dabei zugleich eine spezifische leibgebundene Aneignungspraktik erwerben, die im Folgenden als Praktik des Merkens im Sinne von ,Spüren‘ bezeichnet wird. Diese Bezeichnung greift ethnomethodologisch auf eine Redeweise der Trainerin zurück.

Das erste Beispiel stammt aus der ersten Trainingsstunde mit einer neuen Mädchengruppe. Das Ziel dieser Einstiegsstunde besteht darin, dass die Mädchen lernen, Stimme und Körper zu mobilisieren und als Ressource zur Abwehr eines (männlichen) Aggressors einzusetzen. Diese Aufgabe wird in unterschiedliche Teilaufgaben zerlegt, die zunächst als Einzelkomponenten vermittelt und trainiert und am Ende zum übergeordneten Ganzen einer multimodalen Gestalt verbunden werden. Die Teilaufgaben bestehen aus zu erlernenden Bewegungen, Schreien und kombinierten Laut-Bewegungs-Mustern.

In der folgenden Übungsphase geht es darum, entschlossen einen Schritt nach vorne $\mathrm{zu}$ machen. Als Untereinheit, Vorbedingung und präparatorische Teilkomponente gehört dazu, zunächst einen festen Stand auf dem Boden einzunehmen, bei dem Füße und Beine in eine bestimmte Position gebracht werden. In einer späteren Übungsphase werden Arme und Stimme integriert. Die Analyse fokussiert auf die Verfahren, mittels derer die Trainerin die körperliche Demonstration in die emergierende Verbaläußerung integriert und dergestalt relevant setzt, dass ihre Rezipientinnen verstehen, was sie in welchem Moment wahrnehmen und später in ihr eigenes Körper- und Aktionsschema integrieren sollen. Die instruierende Handlung der Trainerin erstreckt sich von Zeile 1 bis Zeile 4. Auf sie folgt in der 2-sekündigen Pause die instruierte Handlung der Mädchen, die im dritten Schritt von der Trainerin mit geNAU (Z.6) ratifiziert wird: 
Beispiel 1: „sO KURZ da:“

Abb. 1

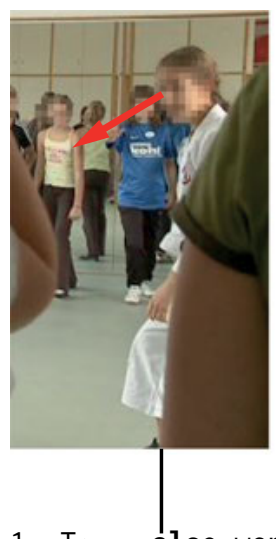

Abb. 2

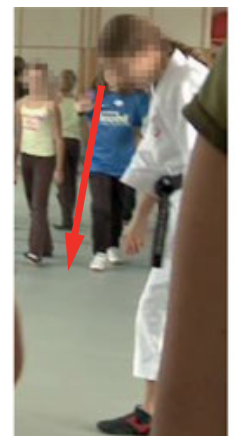

Abb. 3

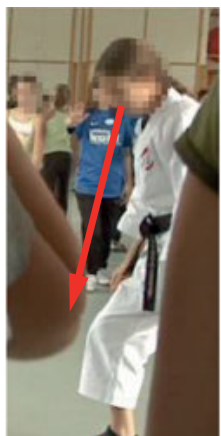

Abb. 4

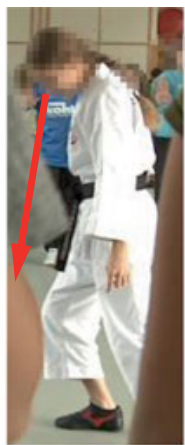

$1 \mathrm{~T}$ : also wenn ihr jetzt MERKT,

2

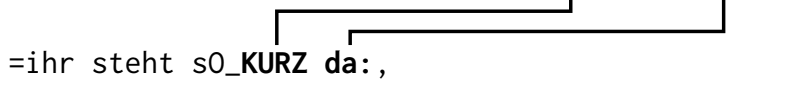

Abb. 5

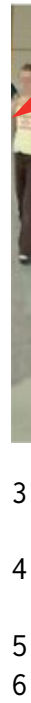

5
Abb. 6

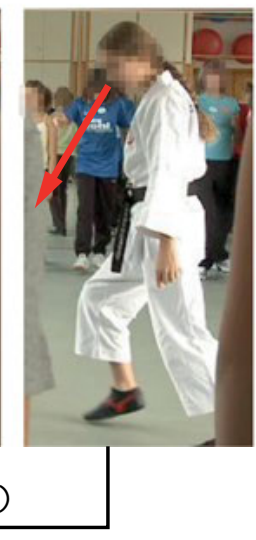

dann macht Einfach n_bIsschen den SCHRITT länger?

(2.0)

geNAU,

$\mathrm{Zu}$ Beginn der Instruktion blickt die Trainerin in die Runde ihrer Adressatinnen (Abb. 1). Im Verlauf der ersten Turnkonstruktionseinheit richtet sie ihre visuelle Aufmerksamkeit ostentativ nach unten auf ihre Füße (Abb. 2). Mit diesem zeigenden Blick (Stukenbrock 2015: 177-192) stellt die Trainerin den relevanten Wahr- 
nehmungsraum für den folgenden Teil der Instruktion her und projiziert weitere Aktivitäten in ihrem leiblichen Umraum.

Diese erste Turnkonstruktionseinheit (turn constructional unit, im Folgenden TCU) besteht aus der Protasis einer Konditionalkonstruktion (Z. 1: wenn ihr jetzt MERKT,), deren Apodosis in Z. 4 folgt (dann macht einfach n_bIsschen den SCHRITT länger?). Die erste TCU endet mit einem Verb der (Selbst-)Wahrnehmung bzw. des Gewahrwerdens (Z. 1: MERKT), das seinerseits einen Objektsatz projiziert, der zwischen Protasis und Apodosis tritt und das Objekt bzw. den Gegenstand des zu Merkenden ausdrückt. Die Protasis (wenn ihr jetzt MERKT,) formuliert nicht nur die Bedingung, unter der der Sachverhalt in der Apodosis eintritt, sondern neben der konditionalen Lesart ergibt sich eine temporale, deiktisch auf die Sprechzeit der emergierenden Äußerung bezogene Lesart (vgl. die Markierung durch das hier allerdings bereits verblasste proximale Temporaldeiktikon jetzt), derzufolge die Mädchen zu einem aktiven Vollzug der in der Protasis formulierten Praktik des Merkens aufgefordert werden. Das, worauf dieses Merken $\mathrm{zu}$ richten sei, wird erst im Objektsatz ausgedrückt.

Über die syntaktische Projektionskraft dieses Verbs verdient dessen Semantik besondere Beachtung. Ich bezeichne es als Verb des Gewahrwerdens, das in Verbindung mit dem - valenzgrammatisch geforderten - Objektperzept als zentrale Komponente ein Moment leiblicher Selbstwahrnehmung beinhaltet, die als solche kognitiv zugänglich gemacht werden kann. Mit anderen Worten ist nicht nur das, was bemerkt wird, als Reflexionsobjekt zugänglich und benennbar, sondern der Prozess, in den das leiblich konstituierte Subjekt aktiv involviert ist und durch den es sich beständig und emergent konstituiert, kann und soll selbst bemerkt werden.

Etymologisch leitet sich das Verb merken aus germanisch *mark-ija mit der Bedeutung ,merken, kennzeichnen“ (Kluge 1989) her. Grimm gibt als lateinische Übersetzungen notare, animadvertere und memoria tenere an und nennt als „die ursprüngliche bedeutung: mit einem zeichen versehen, kenntlich machen“ (DWB online).

Demjenigen, worauf die durch das Verb merken geforderte Objektergänzung referiert, wird durch die Verbsemantik phänomenologisch ein spezifischer Status zugeschrieben: Es handelt sich um eine besondere Art von Perzept, das erstens markiert, mit einem wahrnehmbaren Zeichen versehen, und zweitens dasjenige ist, worauf das leiblich konstituierte, handelnde Subjekt seine äußere und innere Aufmerksamkeit, seinen Geist bzw. seine Seele (anima) richtet. Es stellt einen Gegenstand äußerer und innerer Aufmerksamkeit dar, der sowohl ,außen“, durch oder als etwas Äußeres, als auch ,von innen', durch und als etwas ,Inneres“ wahrzunehmen ist. Diese animadvertere-Praktik eines äußerlichen und innerlichen Gewahrwerdens vollzieht sich als einheitliches, gestalthaftes Ganzes. In die- 
sem Prozess der Wahrnehmung und des Selbst-Gewahrwerdens sind die Mädchen aufgefordert, das in der emergierenden Performanz der Trainerin zu Sehende in einem zwischenleiblichen Akt der Introjektion auf den eigenen Leib zu beziehen.

Im Verlauf der Instruktion wird dasjenige, dessen sich die Mädchen durch die visuelle Wahrnehmung der Aktivitäten der Trainerin am eigenen Leib gewahr werden sollen, weiter erarbeitet. Dabei verändert die Trainerin die Position von Körper und Füßen, indem sie das in Schrittlänge vorangestellte Bein etwas zurücknimmt (Abb. 3). Dieser Moment wird durch das Modaldeiktikon $s O$ (Z.2) projiziert und indiziert. Anschließend weist die Trainerin mit einer Zeigegeste auf den verengten Raum zwischen ihren Füßen (Abb. 4) und richtet auch ihren Blick weiterhin auf den von den Adressatinnen wahrzunehmenden Demonstrationsraum. In der anschließenden Pause blickt sie, während die Körperpositur eingefroren wird, zu ihren Adressatinnen (Abb. 5), die ihre vorherige Position verändern und die neue Schrittlänge ausprobieren.

Die Trainerin beendet das Adressatenmonitoring (Stukenbrock 2015, 2016), indem sie ihren Blick zu Beginn der Apodosis erneut auf den Boden richtet und damit eine körperliche Folgeaktivität projiziert. Anschließend verändert sie synchronisiert mit der verbalen Beschreibung die Position der Füße (Abb. 6). Währenddessen hält sie den Blick nach unten gerichtet. In der unmittelbar folgenden Pause (Abb. 6) reorientiert sie sich auf ihre Adressatinnen, um deren visuelle Orientierung und körperlichen Aktivitäten zu überprüfen, die sie dann ratifiziert (Z. 5: geNAU,). Ihren Erläuterungen zufolge ermöglicht diese Fußposition eine bessere Balance und einen festeren Stand. Indem die Mädchen ihrerseits ihre Position korrigieren, liefern sie der Trainerin ein verkörpertes Verstehensdisplay der Instruktion, die als leibliche Praktik einer festen Standeinnahme nicht nur als knowing that, sondern als knowing how (Ryle 1949, 1971) realisiert werden muss. Der Gebrauch des Modaldeiktikons $S O$ (Z. 2) markiert denjenigen Moment in der leiblichen Performanz der Trainerin, in dem diese mit dem umzustellenden Bein den Boden berührt und dadurch die neu einzunehmende Position markiert (vgl. zur multimodalen Indexfunktion von so Streeck 2002 sowie Stukenbrock 2010, 2015: 416-440).

Wie das zweite Beispiel zeigt, ist das Problem, einen optimalen Stand einzunehmen, nicht trivial, sondern Gegenstand weiterer interaktiver Bearbeitung. Als neuer Aspekt kommt der Bezug auf die Dimensionen des eigenen Leibs hinzu. Maß genommen wird nun nicht mehr nur äußerlich, indem die Mädchen sich visuell an der Trainerin orientieren, sondern indem sie die eigenleiblichen Dimensionen begreifen: 


\section{Beispiel 2: „die füße SO auseinander“}

Abb. 8

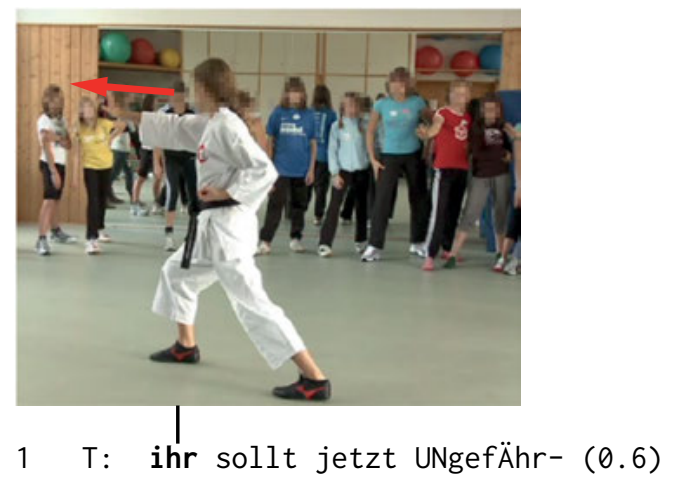

Abb. 9

Abb. 10

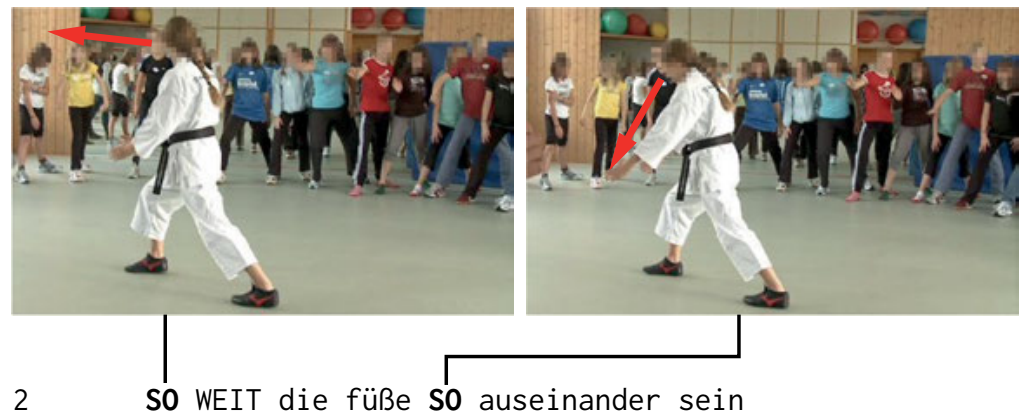

$\mathrm{Zu}$ Beginn der Sequenz befindet sich die Trainerin in der zuvor demonstrierten Position (Schrittposition, rechter Arm zur Abwehr ausgestreckt), ihr Blick ist auf die Adressatinnen gerichtet (Abb. 8). Während in der vorherigen Sequenz der Abstand zwischen vorderem und hinterem Bein erarbeitet wurde, ist nun der seitliche Abstand zwischen linkem und rechtem Fuß in Relation zum eigenen Körper Gegenstand der Demonstration.

Dazu führt die Trainerin zwei Zeigegesten am eigenen Körper aus (Abb. 9) und blickt dann ostentativ nach unten auf ihren durch die Arme begrenzten leiblichen Umraum (Abb. 10). Dabei dient das Modaldeiktikon SO (Z. 2) dazu, exakt die Momente in der emergierenden Instruktion zu markieren, in denen die Adressatinnen den leiblichen Demonstrationen besondere visuelle Aufmerksamkeit widmen sollen. Wie in der vorherigen Sequenz friert die Trainerin die demonstrierte Körperpositur ein, während sie ihren Blick zum Adressatenmonitoring wieder auf die Mädchen richtet (Abb. 11). 
Abb. 11

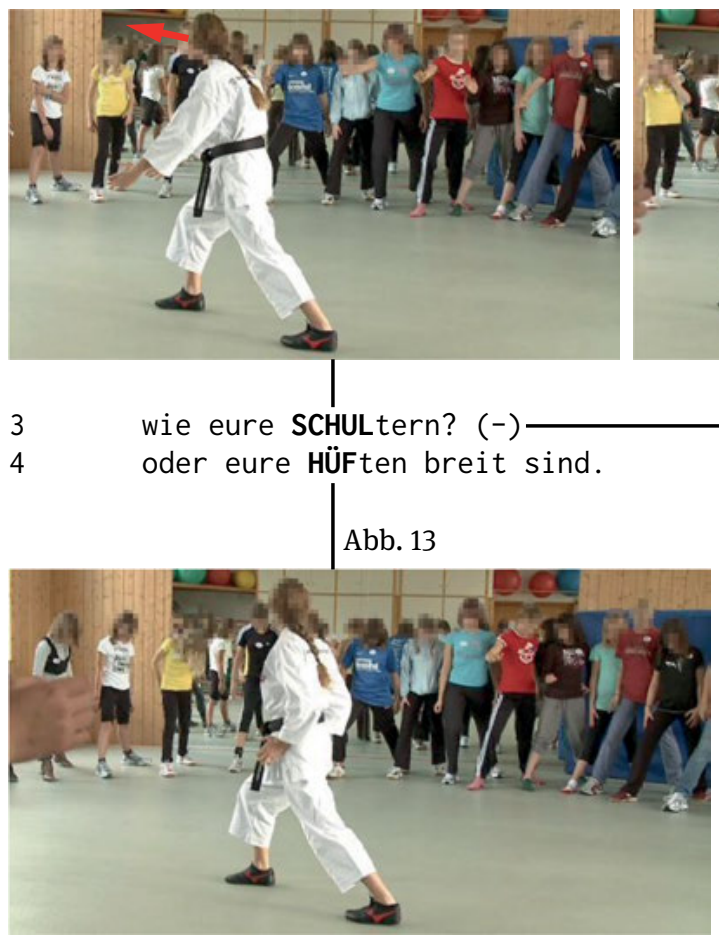

Abb. 12

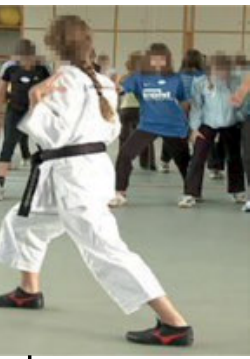

Im nächsten Zug führt sie einen Vergleich durch, der die Aufmerksamkeit der Mädchen auf die Dimensionen des eigenen Leibs mit Schultern und Hüften als maßgebenden Bezugsgrößen richtet. Das Adverbial so, das in der emergierenden Äußerung der Trainerin zunächst als gestisch verwendetes, d.h. eine Sprechergeste benötigendes und damit den Blick der Adressatinnen einforderndes Modaldeiktikon fungiert (vgl. zur Blickeinforderung deiktischer Ausdrücke Stukenbrock 2018a, 2018b, 2018c), wird dadurch retrospektiv als Bestandteil einer Vergleichskonstruktion (Auer 2006) reanalysiert.

Der Vergleich ist zweiteilig organisiert. In einem ersten Schritt werden zunächst die Schultern als Orientierungsgröße eingeführt (Z. 3: wie eure SCHULtern?). Während das Pronomen eure in der Nominalphrase adressatendeiktisch an die Mädchen gerichtet ist, führt die Trainerin eine Zeigegeste am eigenen Körper aus (Abb. 12), der dadurch interaktiv als leibliches Analogon der jeweils eigenleiblichen Dimensionen der Mädchenkörper hergestellt wird. Wie an der visuellen Reorientierung einiger Mädchen in Abb. 12 zu erkennen ist, suchen diese eine Refokussierung auf die eigenleiblichen Relationen nachzuvollziehen, indem sie ein Selbstmonitoring unternehmen. 
Nach einer kurzen Pause setzt die Trainerin den Vergleich mit der disjunktiven Konjunktion oder fort und präsentiert als alternative Bezugsgröße die Hüften (Z. 4: oder eure HÜFten breit sind.). Dabei referiert das Possessivpronomen adressatendeiktisch auf die Mädchen, während die Zeigegesten redebegleitend am eigenen Körper ausgeführt werden (Abb. 13). Im Unterschied zur vorausgehenden, durch so verbaldeiktisch indizierten Demonstration hält die Trainerin ihren Blick nun durchgehend auf die Adressatinnen gerichtet (Abb. 11-13). Auch bei der Herstellung des neuen Zeigeziels blickt sie nicht auf den Gesten- und Wahrnehmungsraum, sondern zu ihren Adressatinnen. Auch wenn beim Zeigen am eigenen Körper ein die Adressatenwahrnehmung steuernder Sprecherblick aufgrund der visuellen Unzugänglichkeit bestimmter Regionen des eigenen Körpers nicht immer möglich ist (Stukenbrock 2008, 2015: 394-416), liegt der Grund für die Blickabstinenz hier nicht an der visuellen Unzugänglichkeit des Zeigeziels (Hüften der Trainerin), sondern gründet vielmehr in der interaktiv herzustellenden Relation zwischen eigenem und fremdem Leib, zwischen demonstrierendem, zeigend-gezeigtem (Trainerin) und aneignendem, inkorporierendem Leib (Mädchen). Indem die Trainerin gerade nicht wie in den vorherigen Sequenzen auf Regionen des eigenen Leibs bzw. dessen Umraum blickt, lädt sie ihre Adressatinnen auch nicht zum gaze following ein. Ziel von deren Aufmerksamkeitsorientierung sind in diesem Fall nicht die Hüften der Trainerin, sondern die Dimensionen des eigenen Körpers. Dies signalisiert das ununterbrochene visuelle Adressatenmonitoring, das die Trainerin im Verlauf ihrer Erläuterungen und Demonstrationen durchführt und das für die Mädchen als Prüfblick erkennbar ist.

Zwischenbilanz: Als Schlüsselressourcen zur Vermittlung zwischen eigenem und fremdem Leib und zur Herstellung einer pädagogischen Zwischenleiblichkeit, die einem durch Fremdwahrnehmung, Nachmachen und Selbstwahrnehmung zu vollziehenden, inkorporierenden Lernen dient, fungieren:

- der Gebrauch des Verbs merken in der Bedeutung äußeren und inneren Gewahrwerdens,

- das Modaldeiktikon SO als Index visuell wahrzunehmender Aktivitäten der Trainerin,

- Blickpraktiken der Trainerin, die als ostentatives Mittel die visuelle Wahrnehmung der Adressatinnen steuern und

- visuelle Monitoringpraktiken der Trainerin, mit denen diese einerseits die visuelle Wahrnehmung ihrer Adressatinnen überprüft und andererseits die korrekte Durchführung der instruierten Handlung überwacht. 


\subsection{Wiederholung, Verkürzung, Reduktion}

Im Folgenden werden Sequenzen untersucht, in denen eine bestimmte Teilbewegung wiederholt wird. Die These lautet, dass Wiederholungen aufgrund des interaktiv aufgebauten gemeinsamen Vollzugswissens multimodale Verdichtungen und Reduktionen ermöglichen, die in der lokalen Interaktionsgeschichte, die die Beteiligten miteinander hervorbringen und gestalten, $\mathrm{zu}$ - ad hoc entstehenden Sedimentierungen führen.

Die Beispiele (3) und (4) illustrieren zwei Instanzen derselben Demonstration. Während bei der erstmaligen Ausführung mehr Ressourcen mobilisiert und ein komplexeres Format verwendet wird, weist bereits die erste Wiederholung Spuren einer Reduktion auf. Die aus Erklärung und Demonstration bestehende Teilinstruktion der Trainerin ist als erster Paarteil gestaltet, der eine von den Schülerinnen unmittelbar auszuführende instruierte Handlung konditionell relevant macht. Sie werden aufgefordert, dem Vorbild der Trainerin folgend die Hände in einer bestimmten Weise an den Hüften zu platzieren.

\section{Beispiel 3: „hÄnde SO in die hÜfte“}

Abb. 14
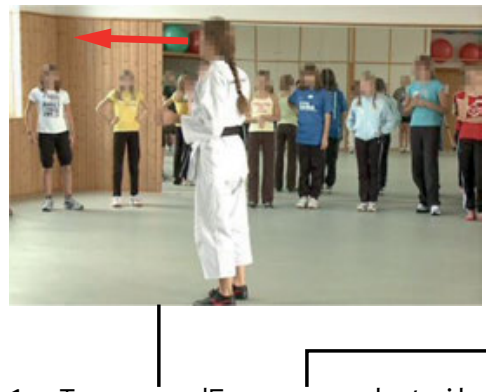

$1 \mathrm{~T}$ : un_dEswegen nehmt ihr jetzt ERSTmal,

Abb. 15

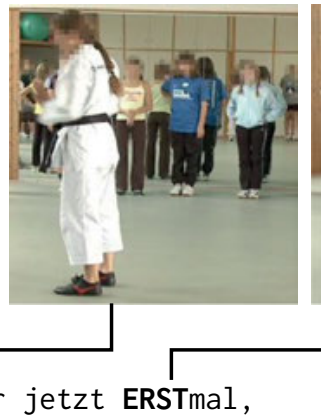

Abb. 16

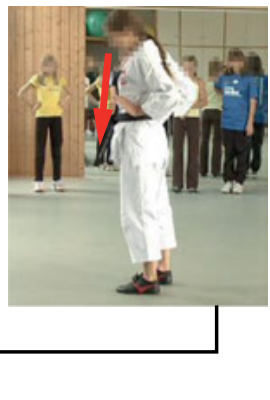

Abb. 17
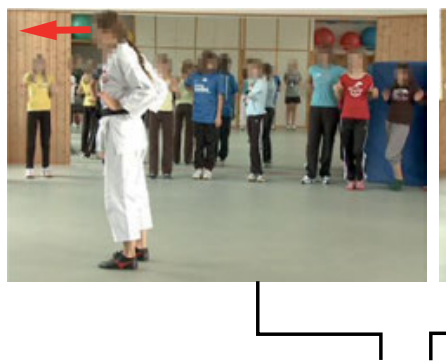

Abb. 18

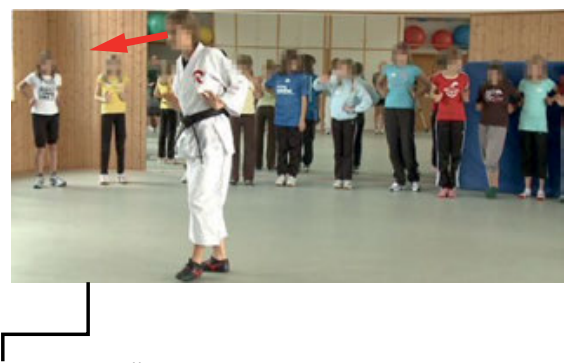

2 die (.) hände So in die HüFte?

$3 \quad(1.0)$

4 T: das mAchen wir später NICHT mehr; 
$\mathrm{Zu}$ Beginn der Sequenz hält die Trainerin ihre Hände in Ruheposition vor dem Körper und richtet den Blick auf die Adressatinnen (Abb. 14). Im Verlauf ihrer Äußerung blickt sie auf ihre Hände, hebt diese mit nach außen weisenden Handflächen leicht an (Abb. 15) und platziert sie oberhalb ihrer Hüften, wobei die Handflächen weiterhin nach außen weisen (Abb. 16). Der Blick folgt der Bewegung der Hände und ist am Ende nach unten auf die eigene Leibesmitte mit den seitlich positionierten Händen gerichtet (Abb. 16). Der ostentativ auf das eigene leibliche Tun orientierte Blick (vgl. zur flag function des Blicks Streeck 2002) in multimodaler Gestalteinheit mit dem Modaldeiktikon SO (Stukenbrock 2010, 2015) indiziert den Adressatinnen die Wichtigkeit des Demonstrierten als visuell Wahrzunehmendem.

Die Relevanz der visuellen Wahrnehmung wird hier also mehrfach kontextualisiert, wie dies bereits in Beispiel (1) zu sehen war. ${ }^{1}$ Anschließend wendet die Trainerin ihren Blick zurück zu den Adressatinnen (Abb. 17). Währenddessen friert sie ihre Körperpositur ein und kontrolliert die Aufmerksamkeitsorientierung der Adressatinnen zu ihrer Linken sowie die Umsetzung ihrer Instruktion. Die stark steigende Intonation am Ende der instruierenden Handlung, die von einer Pause gefolgt und einem Adressatenmonitoring begleitet wird, mobilisiert kein verbales Verstehensdisplay der Adressatinnen, sondern die Ausführung der instruierten Handlung als leibliche Verstehensdokumentation. Das Adressatenmonitoring wird ostentativ in der Pause fortsetzt. Dazu dreht sich die Trainerin mit eingefrorener Gestik um die eigene Achse, um alle im Halbkreis um sie herum platzierten Mädchen in den Blick nehmen zu können. Diese demonstrative visuelle Orientierung auf die Mädchen macht eine unmittelbare Implementierung der Instruktion konditionell relevant, auch wenn sie ein Tun zum Gegenstand hat, das als vorübergehende Hilfskonstruktion markiert wird (Z. 4: das mAchen wir später NICHT mehr;). In der darauffolgenden Wiederholung wird die gleiche Instruktion mit reduzierten multimodalen Ressourcen erteilt, wie das vierte Beispiel zeigt.

\footnotetext{
1 Es zeigt sich, dass die Körperregion der Hüfte durchaus mit dem eigenen Blick aufgesucht werden kann und wird, was das Argument im vorherigen Abschnitt bekräftigt, dass der Verzicht auf einen Gesten- und Wahrnehmungsraum indizierenden Blick nicht auf visuelle Unzugänglichkeit dieser Region des eigenen Körpers zurückzuführen ist, sondern interaktive Gründe hat.
} 


\section{Beispiel 4: „hände so in die HÜFte“}

$1 \mathrm{~T}: \quad<<$ acc >okay nochmal zuRÜCK, >

Abb. 19

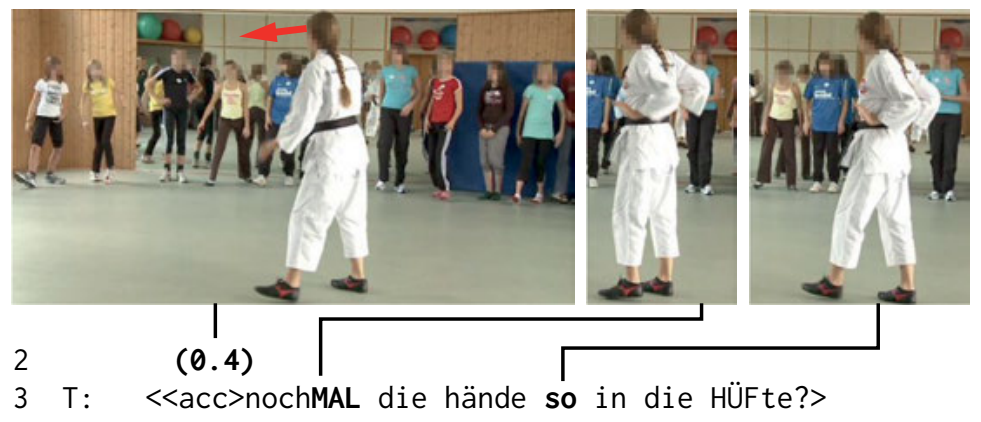

Abb. 22

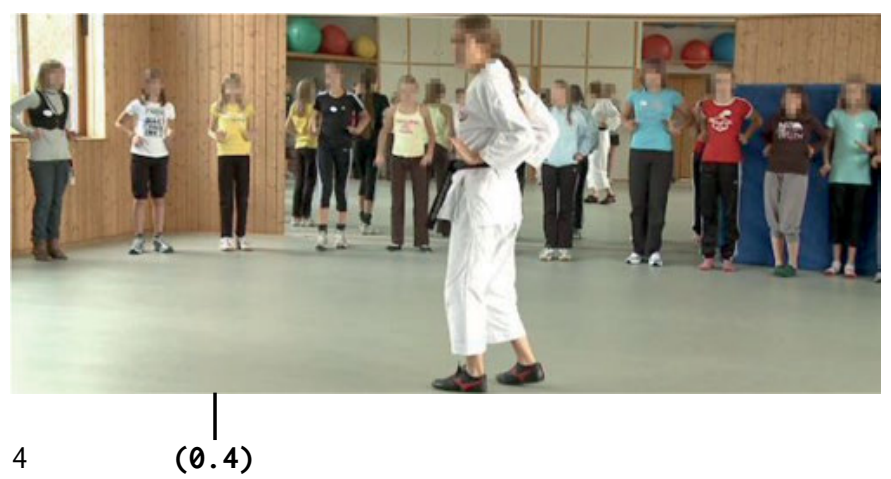

Die Gruppe befindet sich mitten in der Übungsphase. Die Trainerin rearrangiert die Gruppe, indem sie sie auffordert, einen Schritt zurückzumachen (Z. 1). Dies vollziehen die Mädchen in der Pause (Z. 2), während die Trainerin sie ostentativ in den Blick nimmt (Abb. 19). Die interaktionsräumliche Rekonfiguration ist die Voraussetzung dafür, dass die Trainerin mit der nächsten Teilinstruktion fortfahren kann. Diese stellt eine Wiederholung der vorherigen Instruktion (Beispiel 3) dar. Allerdings setzt die Trainerin ihren Blick nun nicht mehr als visuellen Index ihrer Gesten ein, sondern richtet ihn kontinuierlich auf die Mädchen (Abb. 20-22). In der anschließenden Pause wird das Adressatenmonitoring, diesmal nur mit einer Kopf- anstelle einer Körperdrehung, fortgesetzt (Abb. 22).

Es sind noch weitere Reduktionen des multimodalen Formats festzustellen. Nicht nur fehlt der zeigende Blick; zudem ist das Adverbial so reduziert, es trägt keinen Fokusakzent mehr. Gesten und instruktionsrelevante Körperpartien wer- 
den nicht mehr durch ein gestisch gebrauchtes, prosodisch markiertes Modaldeiktikon und einen zeigenden Blick als visuell Wahrzunehmendes mehrfach markiert. Der multimodale Verdichtungsraum (Stukenbrock 2008: 12) ist deutlich reduziert.

Indem die Trainerin die Instruktion mit dem akzentuierten Temporaladverb nochMAL (Z. 3) beginnt, rahmt sie das Kommende als Wiederholung von etwas, das bereits im common ground verankert ist. Die Mädchen können also schon zu Beginn der Äußerung antizipieren, dass etwas Bekanntes demonstriert wird, und eine entsprechende Haltung im Hinblick auf die Aktivitäten der Trainerin und die von ihnen erwarteten Folgehandlungen einnehmen.

Der Wiederholungscharakter dieser Sequenz zeigt sich darin, dass die zu ihrer Durchführung mobilisierten Ressourcen reduziert sind (Wegfall des ostentativen Blicks mit flag function nach Streeck 2002, Wegfall des Fokusakzents beim Adverbial so, Kopf- statt Körperdrehung beim Adressatenmonitoring). Auch der syntaktische Aufwand ist geringer, denn statt der zwei Turnkonstruktionseinheiten im dritten Beispiel (Z. 1-2) benötigt die Trainerin im vierten Beispiel nur eine (Z. 3), die zudem mit erhöhter Sprechgeschwindigkeit gesprochen wird.

Zwischenbilanz: Die Analyse der Beispiele (3) und (4) belegt den zu Beginn dieses Abschnitts postulierten Prozess einer in und durch Wiederholung erfolgenden Reduktion, die zugleich eine temporale Verdichtung und Akzeleration ermöglicht. Dies manifestiert sich in Beispiel (4) nicht nur in der beschleunigten Sprechgeschwindigkeit der Trainerin, sondern auch im reduzierten syntaktischen Format. Der Aspekt der Beschleunigung und Dynamisierung wird in Abschnitt 3.4 wieder aufgegriffen. Zuvor soll allerdings der besondere Fall der Meta-Instruktion diskutiert werden, der als markierter Fall qua Kontrast ein Licht auf Verdichtungsund Reduktionsprozesse wirft.

\subsection{Meta-Instruktionen}

Nachdem im vorherigen Abschnitt reduzierte Varianten dargestellt wurden (Beispiel 4), die sich aus einer sequenziellen Wiederholung ergaben, wird im Folgenden der entgegengesetzte Fall erhöhter Komplexität untersucht. In den bisherigen Sequenzen folgten instruierende und instruierte Handlung ohne explizite Anweisung unmittelbar aufeinander. Demgegenüber gibt die Trainerin im nächsten Beispiel zusätzlich eine entsprechende Meta-Instruktion. An die Stelle der Inferenz, dass der instruierenden Handlung als erstem Paarteil unmittelbar eine instruierte Handlung als zweitem Paarteil folgen soll, tritt eine explizite Anweisung. 
Meta-Instruktionen fügen der Reflexivität und Indexikalität verbaler Interaktion eine zusätzliche Reflexionsebene hinzu. Dies geschieht, wenn Interaktionsbeteiligte annehmen, dass ein Verstehen „for all practical purposes“ (Garfinkel 1967) gefährdet sein könnte, da Kontextualisierungshinweise nicht ausreichend, ambig oder widersprüchlich sind.

In der folgenden Instruktion, die die Art der Ausführung einer körperlichen Handlung betrifft, wird durch die eingeschobene Meta-Instruktion verdeutlicht, dass als Folgehandlung eine körperliche Ausführung erwartet wird. Gegenstandsinstruktion (auf die Ausführungsart der Bewegung bezogene Instruktion) und Meta-Instruktion (auf die Gegenstandsinstruktion bezogene Instruktion) bilden als komplexe Instruktionshandlung den ersten Paarteil, deren zweiter Teil von den Adressatinnen leiblich zu vollziehen ist. Anders als in den bisher betrachteten Instruktionen kommt die Trainerin hier nicht ohne eine explizite Aufforderung zum Nachmachen aus.

\section{Beispiel 5: „einmal AUSprobieren“}

1 T: <<acc>aber wenn ihr jetzt HIER,

$2 \quad={ }^{\circ} \mathrm{h}$ mit TURNschläppchen oder nachher dann auch

3 mit Socken an polster tretet,>

Abb. 23

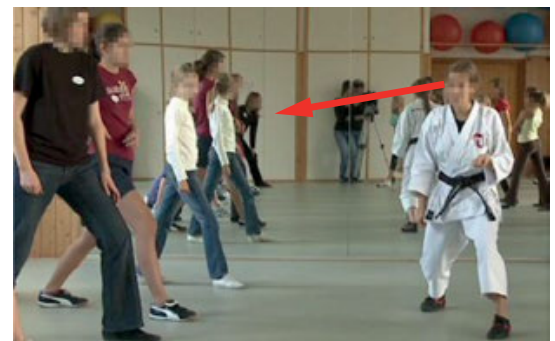

4

5

6

ist es !GANZ! WICHtig, =dass $\mathrm{ihr}$ NICHT? ${ }^{\circ} \mathrm{h}$ (.)

einmal AUUSprobieren,
Abb. 24

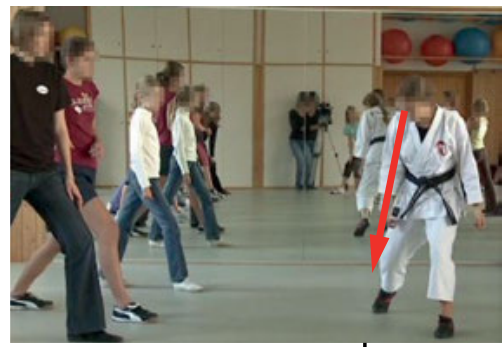


Abb. 25

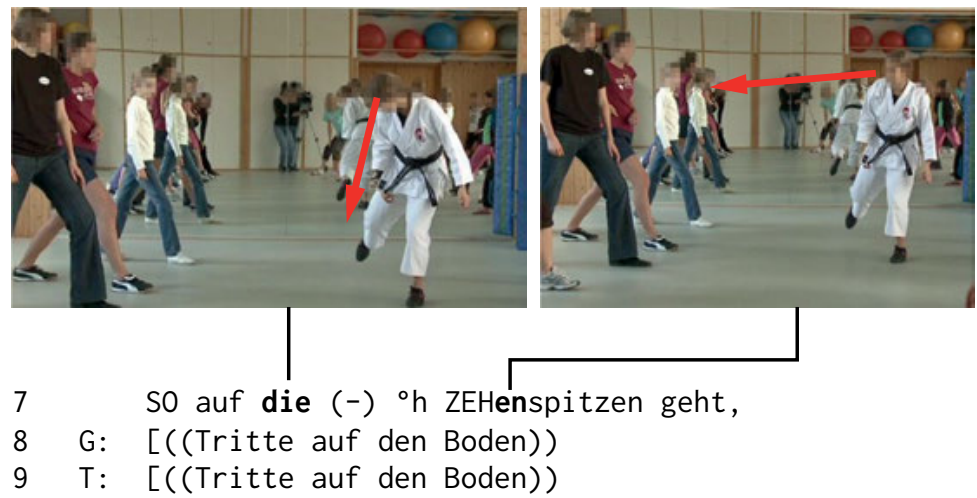

Die instruierende Handlung der Trainerin erstreckt sich von Z. 1 bis Z. 7. Wie im ersten Beispiel verwendet sie eine Konditionalkonstruktion, deren Protasis (Z. 1-3) die Bedingungen nennt, unter denen der Sachverhalt in der Apodosis (Z. 5-7) zutrifft. Die Meta-Instruktion, bestehend aus einer deontischen Infinitivkonstruktion (vgl. Deppermann 2006) ist syntaktisch als Parenthese (Z. 6: einmal AUSprobieren,) in die Struktur der Konditionalkonstruktion eingebettet. Wie in Beispiel 1 kommt zur konditionalen eine temporale Bedeutung hinzu, die hier auch durch die Temporaladverbien jetzt (Z. 1) und nachher (Z. 2) ausgedrückt wird. Die Apodosis ist ihrerseits mehrgliedrig. Sie besteht aus einem Kopula-Satz und einem mit dass eingeleiteten Objektsatz (Z. 5: dass ihr NICHT? und Z. 7: SO auf die (-) ${ }^{\circ} \mathrm{h} \mathrm{ZE}$ Henspitzen geht). Dieser emergierende Objektsatz wird suspendiert, um die MetaInstruktion (Z. 6: einmal AUSprobieren,) an einem Punkt einzufügen, an dem das akzentuierte Negationsadverb NICHT durch die exponierte Position vor der linken Parentheseklammer (Schönherr 1993) besonders in den Vordergrund rückt. Aufgrund der unabgeschlossenen syntaktischen Gestalt und den uneingelösten Projektionen herrscht eine starke Fortsetzungserwartung, die durch die Negation noch zusätzlich Gewicht erhält: Im Folgenden ist offenbar eine Warnung zu erwarten. In der parenthetisch eingefügten Meta-Instruktion fordert die Trainerin die Mädchen ausgerechnet an dieser Stelle explizit auf, das Demonstrierte nachzumachen. Warum tut sie das, nachdem sie in den vorherigen Sequenzen darauf verzichtet hat?

Das Warum-Problem ist untrennbar mit dem Problem des Wann verbunden. Es gibt mehrere Gründe, warum sich die Trainerin an dieser Stelle veranlasst sieht, die Schülerinnen explizit zu instruieren, die Bewegung nachzumachen. Erstens demonstriert sie, wie man die zu übende Bewegung gerade nicht machen soll. Sie erteilt eine negative Instruktion, die sich auf ein Detail der auszuführenden Bewegung bezieht, das die Mädchen beachten sollen, um Schmerz zu 
vermeiden und Verletzungsgefahr abzuwenden. Die dadurch aufgebaute konditionelle Relevanz besteht darin, die demonstrierte Bewegung künftig zu vermeiden. Verstehen und compliance werden folglich durch Unterlassen demonstriert. Demgegenüber kann eine Ausführung als Dokumentation von Nicht-Verstehen oder non-compliance verstanden werden. Da die Trainerin nichtsdestotrotz an dieser Stelle will, dass die Schülerinnen am eigenen Leib ein - gleichwohl abgepuffertes - Gefühl für die falsche, Schmerz erzeugende Bewegung bekommen, fordert sie sie zum Ausprobieren in abgebremster Form auf.

Zweitens hängt die parenthetische Aufforderung mit der syntaktischen Formatierung und interaktiven Kontextualisierung eines größeren Pakets (big package) zusammen. Der Zeitpunkt, zu dem das in diesem Augenblick Vermittelte relevant wird, ist ein späterer: Z. 1-2. Die Instruktion besitzt folglich einen größeren Skopus als die vorherigen Teilinstruktionen. Sie bezieht sich nicht nur auf die aktuelle, sondern auch auf spätere Übungsphasen (Z. 2-3: nachher dann auch mit soCken).

Neben dem aufwändigen syntaktischen Format werden für die instruierende Handlung eine Reihe weiterer Ressourcen mobilisiert: So setzt die Trainerin, nachdem sie zunächst auf die Mädchen orientiert ist (Abb. 23), ihren Blick zum Zeigen auf ihren hinteren Fuß ein (Abb. 24), als sie die parenthetische MetaInstruktion (Z. 6) formuliert. Neben dem Blick als - im wörtlichen Sinn - vorausschauender Ressource wird die leibliche Demonstration durch das akzentuierte Modaldeiktikon $S O$ (Z. 7) projiziert. Es markiert temporal die unmittelbar darauf einsetzenden Auf- und Abbewegungen des Fußes (Abb. 25), die die Mädchen dann mit hörbarem Klopfen ausführen (Z. 8). Die Trainerin setzt ihrerseits die Bewegung für einen kurzen Moment im Chor mit den Mädchen fort, wobei sie den Blick wieder auf ihre Adressatinnen richtet. Im Unterschied zum reduzierten Format im vorherigen Abschnitt wird hier nicht nur eine komplexe syntaktische Konstruktion mit einer als Parenthese eingeschobenen Meta-Instruktion verwendet. Das Blickmuster ist ebenfalls aufwändiger gestaltet: Die Trainerin wechselt zwischen der Orientierung auf den eigenen Fuß zur Projektion und Ostension des Demonstrationsraums und dem Adressatenmonitoring hin und her. Während in Beispiel 4 neben der Akzeleration durch Reduktion auch eine Beschleunigung der Sprechgeschwindigkeit zu beobachten war, treten hier im Verlauf der Apodosis Pausen auf, die eine Verzögerung bewirken. Diese ist insofern ikonisch, als die Mädchen die demonstrierte Bewegung nicht voll ausführen, sondern in abgebremster Form mit reduzierter Dynamik und Kraft lediglich simulieren sollen.

Dieses Beispiel illustriert die zentrale Rolle der Temporalität für die interaktive Konstruktion der Bedeutung einer Handlung. Bestandteile des im gemeinsamen Verständigungsprozess herzustellenden Wissens sind nicht nur wasund wie-Komponenten des Wissens (knowing that und knowing how, vgl. Ryle 
1971), sondern auch zeitliche Aspekte des wann. Form, Qualität und Funktion von Instruktionssequenzen reflektieren nicht nur den jeweiligen Instruktionsgegenstand, den lokalen Interaktionskontext und den augenblicklichen Stand der Interaktionsgeschichte der Beteiligten, sondern sie weisen spezifische Temporalitätsstrukturen auf, aus denen sie einerseits emergieren und die sie andererseits hervorbringen. Am deutlichsten wird dies im Übergang von der Instruktion zum Drill, der im letzten Abschnitt untersucht wird.

\subsection{Von der Instruktion zum Drill}

In diesem Abschnitt wird analysiert, wie sich Qualität, Form und Funktion von Instruktionssequenzen im Verlauf der Interaktionsgeschichte der Beteiligten verändern. Die These lautet, dass im Zuge eines mikrointeraktionsgeschichtlichen Aufbaus gemeinsamen Wissens die multimodale Komplexität der Sequenzen durch Wiederholung abgeschliffen, reduziert und zeitlich komprimiert wird. Sequenzen aus Instruktionen mit propositionalem Gehalt und instruierten Folgehandlungen, die im dritten Zug von der Trainerin bewertet bzw. korrigiert werden, werden zu immer rascheren Abfolgen von erster und zweiter Handlung verkürzt. Während sich die Aktivität damit zum sportlichen Drill - der raschen Wiederholung der immer gleichen Abläufe zu Trainingszwecken - entwickelt, verschiebt sich das Lernziel von der korrekten zur möglichst raschen, dynamischen und automatisierten Ausführung der instruierten Handlung. Das im Interaktionsprozess aufgebaute Wissen umfasst unterschiedliche Dimensionen:

- den Instruktionsgegenstand als kognitiv zu erfassendes Phänomen,

- die leiblichen Praktiken seiner Umsetzung als visuell am Anderen wahrgenommenes und eigenleiblich in Aktion erfahrenes Phänomen,

- damit einhergehend ein zunehmendes, motorisch vermitteltes Bewusstsein für die Potenziale und Grenzen der eigenen Aneignungsfähigkeit,

- die Praxis der Lehr-Lern-Interaktion mit der Trainerin und des chorisch-synchronisierten Übens mit und in der Gruppe.

Die später auftretenden Instruktionssequenzen weisen nicht nur Merkmale einer verstärkten Routinisierung auf, die im Verlauf eines ständigen Wechsels zwischen instruierender und instruierter Handlung beim Üben entsteht und beide Paarteile betrifft. Darüber hinaus verändert sich auch die Temporalitätsbeziehung zwischen instruierender und instruierter Handlung, die sich immer stärker auf das Format des „Drills“ hin entwickelt. Die Analysen beginnen mit der komplexesten Sequenz, die zudem eine Meta-Instruktion enthält. 


\section{Beispiel 6: „ich klAtsch in die HÄNde“ (MM_B1_00:13:30)}

01 T: jetzt machen_wa_s So,

02 Ich klAtsch in die HÄNde,

03 und Ihr geht Einfach diesen schrItt nach VORne? (-)

04 ACHtet drauf,

05 dass ihr (--) BREIT genug seid?

06 <<acc>also nIcht wie_n SEILtanz macht,> (-)

07 und vorne im KNIE steht;

08 <<acc>und dann GUCken wir mal-

09 was man nOch so alles (.) FALSCH machen kann;

10 UND->

11 ((klatscht))

$12 \mathrm{G}$ : ((machen Schritt nach vorne))

$13 \mathrm{~T}:<<\mathrm{p}>\mathrm{OKAY} ;>$

In dieser Sequenz gibt die Trainerin eine Meta-Instruktion, die in einem ersten Schritt das Händeklatschen als interaktive Ressource etabliert, das die Funktion des Timings übernehmen und künftig die interpersonelle Koordinierung eines synchronisierten Praktizierens in der Gruppe organisieren soll. Das Händeklatschen fungiert als go ahead oder Startschuss, als gestisch gebrauchtes Zeitdeiktikon, das temporal auf den Moment seiner eigenen Ausführung ,zeigt‘. Interessanterweise starten die Mädchen nie simultan mit dem Klatschen, weder beim ersten Mal noch in den späteren Sequenzen, sondern immer erst unmittelbar darauf. Die Meta-Instruktion wird ihrerseits durch eine metapragmatische Handlung projiziert: jetzt machen_wa_s SO, (Z. 01), Diese Ankündigung macht im Format einer sog. Vorlaufkonstruktion (Auer 2006) ein größeres Paket erwartbar.

Auf die Meta-Instruktion, die das Händeklatschen als gemeinsames Signal desjenigen Moments etabliert, in dem die Mädchen die instruierte Handlung ausführen sollen, folgt die eigentliche Instruktion. Diese ist komplex aufgebaut: Die Trainerin gibt Detailinstruktionen in Form von Erläuterungen und einer parenthetischen ex negativo-Reformulierung (Z. 06), die bei der Ausführung zu berücksichtigen sind. Anschließend liefert sie eine Vorausschau auf spätere Übungsphasen, die dem Üben und der Fehlerkorrektur dienen (Z. 08-09). Die Vorausschau fungiert zugleich als account: Indem die Trainerin Fehler antizipiert, begründet sie implizit die Notwendigkeit künftiger Wiederholungs- und Korrektursequenzen.

Das Händeklatschen (Z. 11), das die Trainerin mit einer weit ausholenden, bühnenhaft inszenierten Bewegung vollzieht, wird durch die akzentuierte Konjunktion $U N D$ (Z.10) projiziert. Unmittelbar darauf führen die Mädchen die instruierte Handlung (Z. 12) aus. Die Sequenz wird durch die Ratifikation der Trainerin abgeschlossen (Z. 13: oKAY;). 
Die sequenzielle Struktur lässt sich wie folgt zusammenfassen: Ein komplexer, multi-unit turn der Trainerin bildet den ersten Paarteil, der als zweiten Paarteil eine chorisch ausgeführte instruierte Handlung der Mädchen konditionell relevant macht. Während letztere ausschließlich körperlich ausgeführt wird, weist erstere ein komplexes Format auf:

1. Position: komplexe Instruktion der Trainerin

- erste Ankündigung durch die Vorlaufkonstruktion mit so (Z. 01)

- Meta-Instruktion (Z. 02)

- Instruktion mit Erläuterungen (Z. 03-07) und negativer Parenthese (Z. 06)

- Ausblick auf Post-Evaluation der Performanz und auf spätere Übungsphasen (Z. 08)

- zweite Ankündigung durch akzentuiertes UND mit gleichbleibender Intonationskontur (Z. 10)

- Klatschen (Z.11).

2. Position: Ausführung der instruierten Handlung durch die Mädchen

3. Position: Ratifikation durch die Trainerin.

Das siebte Beispiel folgt im Handlungsablauf des Trainings unmittelbar auf das sechste Beispiel. Erneut fungiert das Klatschen als Startsignal für die Gruppe:

\section{Beispiel 7: „auf_s klAtschen“(MM_B1_00:14:38)}

$01 \mathrm{~T}$ : OKAY;

02 nochmal zuRÜCK?

$03 \mathrm{G}:$ ((treten zurück))

$04 \mathrm{~T}$ : und JETZT geht ihr auf_s klatschen mit dem Andern bein vor;

$05 \mathrm{~T}: \mathrm{UND}=((\mathrm{klatsch}$ in die Hände $))$

$06 \mathrm{G}:$ ((treten nach vorne))

$07 \mathrm{~T}$ : OKAY;

In dieser Sequenz sind bereits eine erste Reduktion und Komprimierung festzustellen. Die metapragmatische Thematisierung des Klatschens in seiner Funktion als Startsignal für die Ausführung der instruierten Handlung, die in der vorherigen Sequenz eine vollständige syntaktische Einheit bildete, an die sich die eigentliche Instruktion anschloss, wird nun zu einer Präpositionalphrase (Z. 04: auf_s klatschen) verdichtet und in die Instruktion eingebettet (Z. 04: und JETZT geht ihr auf_s klatschen mit dem Andern bein vor;). Wie im sechsten Beispiel wird das Klatschen anschließend durch die Konjunktion UND (Z. 05) projiziert und sehr schnell angeschlossen. Die Mädchen vollziehen die instruierte Handlung (Z. 06), woraufhin die Trainerin die Sequenz durch die Ratifikation oKAY (Z. 07) abschließt. 
Auch im achten Beispiel schickt die Trainerin eine auf das Klatschen bezogene meta-pragmatische Rahmung voraus (Z. 01). Durch das Modaladverb wieder wird die Praktik als bereits bekannt markiert. Während die meta-pragmatische Thematisierung und die Praktik des Klatschens als bekannt etabliert sind, kommt als Gegenstand der instruierten Handlung ein neues Element hinzu (Z. 02: und ihr NEHMT (-) den ARM mit dazu;):

\section{Beispiel 8: „ich KLATSCH wieder“ (MM_B1_00:15:34)}

$01 \mathrm{~T}$ : ich KLATSCH wieder,

02 und ihr NEHMT (-) den ARM mit dazu;

03 ((klatscht in die Hände))

$04 \mathrm{G}$ : ((treten nach vorne und heben Arm))

$05 \mathrm{~T}$ : oKAY;

In dieser Sequenz wird die Konjunktion als das Klatschen projizierendes Element weggelassen. Die Trainerin klatscht unmittelbar nach der Instruktion (Z. 02) in die Hände (Z. 03), die Mädchen führen die instruierte Handlung aus (Z. 04), woraufhin die Trainerin die Ausführung mit oKAY; (Z. 05) ratifiziert. Die Auslassung der Konjunktion führt zu einer weiteren temporalen Kompression.

In der neunten Sequenz führt die Trainerin zunächst eine Korrektur (Z. 01) durch. In unmittelbarem Anschluss daran formuliert sie eine Teilinstruktion, die sich auf die Herstellung der körperlichen Ausgangsposition für die zu übende Bewegung bezieht. Dies implikatiert, dass unmittelbar darauf die Armbewegung erneut praktiziert wird. Im Unterschied zu den vorherigen Sequenzen wird nun die metapragmatische Rahmung weggelassen. Das Händeklatschen wird wie zuvor durch die Konjunktion U:ND (Z. 04) projiziert, das Klatschen wird von einer onomatopoetischen Vokalisierung begleitet (Z. 04: ZACK;), die einen höheren Grad an Dynamik und Schnelligkeit in der Bewegungsausführung kontextualisiert und die Mädchen zu einer forcierten Vorwärtsbewegung anfeuert.

\section{Beispiel 9: „nur EIN ARM“ (MM_B1_00:15:38)}

$01 \mathrm{~T}$ : IMmer nur EIN ARM;

02 NoCHmal (.) HINten in die HÜFte,

$03 \mathrm{G}$ : ((tun Arme und Hände zurück an die Hüfte))

04 T: U:ND [ZACK;

\section{$[((k l a t s c h t$ in die HÄnde $))$}

$05 \mathrm{G}$ : ((treten vor, heben einen Arm))

$06 \mathrm{~T}$ : oKAY;

07 wieder zuRÜCK? 
Diese Übung wird unmittelbar darauf wiederholt. Es folgt eine längere Erklärsequenz, nach der die Übung im selben Format wiederholt wird. In beiden Fällen weist der instruierende Turn der Trainerin das Format $U N D$ + Händeklatschen mit simultaner Vokalisierung ZACK auf.

Nach Beendigung der Abwehrübung mittels Arm wird die nächste Teilkomponente integriert: das Schreien, das die Trainerin als letztes noch fehlendes Element ankündigt. Für die folgende Instruktion wählt sie ein komplexeres Format, indem sie die neue Übungssequenz wieder mit einer metapragmatischen Formulierung einleitet. Das komplexere Format der instruierenden Handlung reflektiert den höheren Aufwand der instruierten Handlung. Für die zu übende Abwehr-Praktik müssen die Mädchen zusätzlich zu den körperlichen Ressourcen ihre Stimme mobilisieren und simultan zu den Körperbewegungen einen lauten NEIN-Schrei produzieren:

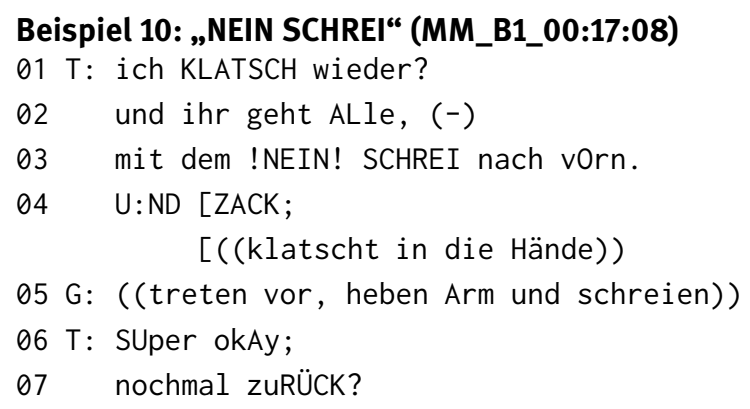

Die metapragmatische Rahmung (Z. 01) stellt eine syntaktisch-prosodische Einheit dar, auf die eine in zwei Intonationseinheiten (Z. 02-03) gegliederte Instruktion folgt. Letztere sind durch eine kurze Pause getrennt. Da die syntaktische Gestalt vor der Pause (Z. 02) noch nicht geschlossen ist, erhält der projizierte zweite Teil (Z. 03) ein starkes Gewicht, wodurch die neue Komponente, die Integration des Nein-Schreis, in den Fokus gerückt wird.

Anschließend leitet die Konjunktion $U: N D$ (Z. 04) das Händeklatschen ein, das von der Vokalisierung ZACK (Z. 04) begleitet wird. Die Mädchen führen die instruierte Handlung mitsamt der neuen Komponente aus, was die Trainerin dieses Mal nicht nur mit $o k A y$, ratifiziert, sondern zusätzlich mit dem Adjektiv SUper (Z. 06) besonders positiv bewertet.

Mit der anschließenden Teilinstruktion nochmal zuRÜCK? (Z. 07) fordert sie die Mädchen auf, in die Ausgangsposition zurückzukehren, und projiziert eine Wiederholungsrunde. 
Auf den ersten Versuch einer simultanen Ausführung von Vorwärtsschritt, Abwehrbewegung und Nein-Schrei folgt ein kurzer Kommentar zur Lautstärke des Schreis, bevor die Trainerin eine zweite Übungssequenz initiiert:

\section{Beispiel 11: „U:ND ZACK“(MM_B1_00:17:30) \\ 01 T: U:ND [ZACK; [( (klatscht in die Hände)) \\ $02 \mathrm{G}$ : ((treten vor, heben Arm und schreien)) \\ $03 \mathrm{~T}$ : nochmal zuRÜCK?}

Die Trainerin verzichtet auf den metapragmatischen Vorlauf. Konjunktion, Vokalisierung und simultanes Händeklatschen konstituieren ein reduziertes, zeitlich verdichtetes Instruktionsformat. Dabei manifestiert sich die zeitliche Verdichtung und Kompression auch im Übergang zwischen den einzelnen Versuchen. Anstelle einer Ratifikation schließt die Trainerin nun direkt die Teilinstruktion nochmal zuRÜCK? (Z. 03) an.

Selbst wenn Kurzkommentare zu Ausführungsdetails eingeschoben werden, rekurriert die Trainerin anschließend auf das fortschreitend sedimentierte Format aus Konjunktion + Vokalisierung mit simultanem Händeklatschen:

\section{Beispiel 12: „U:::ND ZACK“ (MM_B1_00:17:40) \\ $01 \mathrm{~T}: \mathrm{U}::: \mathrm{ND}(0.7)$ [ZACK; $[((k l a t s c h t$ in die Hände $))$}

$02 \mathrm{G}$ : ((treten vor, heben Arm und schreien))

Im zwölften Beispiel tritt, bedingt durch den kurzzeitigen Verlust eines gemeinsamen Aufmerksamkeitsfokus, eine Pause zwischen Konjunktion und Folgekomponenten. Die Pause dokumentiert die kontextsensitive Adaptabilität des Formats, ohne dass die Drillqualität der extrem verkürzten Instruktion verloren ginge. Die temporale Flexibilität bestätigt die Kohäsion zwischen den Teilkomponenten innerhalb des mikrointeraktionsgeschichtlich bereits sedimentierten Formats. Die Projektion der Konjunktion auf die hier formatspezifisch zu erwartenden Folgekomponenten (Vokalisierung mit simultanem Händeklatschen als Startschuss für die chorische Ausführung der Instruktion) ist stark genug, um ohne Retraktion über die Pause hinweg zu wirken. Dabei fungiert die Pause selbst zur Wiederherstellung fokussierter Interaktion (Goodwin 1980, 1981).

Die durch den lokalen Kontext bedingte zeitliche Zerdehnung widerspricht daher nicht dem Argument einer schrittweisen Reduktion, Kompression und zeit- 
lichen Verdichtung der mobilisierten Ressourcen. Im Gegenteil sind Reduktion und temporale Verdichtung als Ergebnis eines mikrointeraktionsgeschichtlichen Sedimentierungsprozesses des entsprechenden Formats die Voraussetzung seines flexiblen, kontextsensitiven Gebrauchs.

In der Folge kommen weitere Reduktionen dadurch zustande, dass singuläres Händeklatschen ohne Mobilisierung anderer Ressourcen als erste Handlung zur Initiierung der chorischen Folgehandlung eingesetzt werden kann:

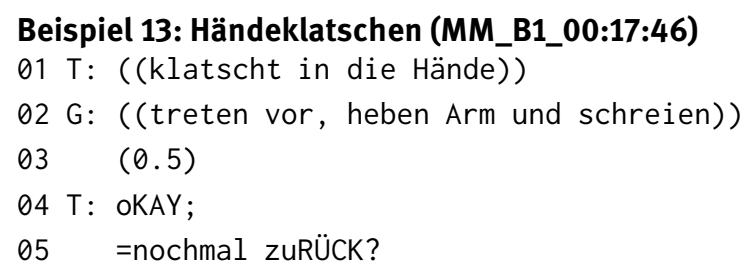

Ziel der wiederholten Einübung immer gleicher Bewegungsabläufe ist eine Automatisierung von Bewegungsmustern, die den Übenden „in Fleisch und Blut übergehen“ sollen. Dabei wirken die Zielqualitäten des Instruktionsgegenstands zurück auf die Praktiken seiner Vermittlung und Aneignung. Diese Reflexivität betrifft instruierende und instruierte Handlung sowie die sich verändernde temporale Relation zwischen den Paarteilen.

So führt wiederholtes Üben zu schnelleren, flüssigeren Bewegungsabläufen der zu erwerbenden Verteidigungspraktiken, die dadurch, dass sie von der Trainerin in immer rascherer Folge eingefordert und von den Mädchen ausgeführt werden, an Tempo, Schlagkraft und Dynamik gewinnen und routinisiert werden. Reaktionsschnelligkeit als Schlüsselmoment erfolgreicher Selbstverteidigung wird damit durch die stetig reduzierte Zeitspanne zwischen instruierender und instruierter Handlung mit eingeübt. Im geschützten Raum der Turnhalle, in dem Angriff simuliert und Verteidigung geübt wird, steht die temporal auf einen einzigen Moment (den Klatsch-Moment) kondensierte erste Handlung der Trainerin nicht nur metonymisch für die immer weiter kondensierte Instruktion, sondern auch metaphorisch für einen blitzschnell erfolgenden Angriff, auf den im Ernstfall unmittelbar, quasi reflexartig, mit einem Maximum an Tempo, Dynamik und Schlagkraft reagiert werden muss. 


\section{Diskussion}

In diesem Beitrag wurden Instruktionssequenzen untersucht, deren Vermittlungsgegenstand Praktiken der Selbstverteidigung sind. Das Grundformat dieser Sequenzen besteht aus einer multimodalen Instruktion der Trainerin, die den ersten Paarteil bildet und als zweiten Paarteil eine von den Schülerinnen chorisch vollzogene instruierte Handlung konditionell relevant macht. Dabei dient der Vollzug der instruierten Handlung nicht nur sequenziell als Verstehensdisplay der Schülerinnen, sondern auch als Ausweis der leiblichen Beherrschung einer Verteidigungspraktik, die von der Trainerin mit professionellem Blick (Goodwin 1994) überprüft, im dritten Schritt ratifiziert oder korrigiert und in Wiederholungssequenzen routinisiert wird.

Trainerin und Schülerinnen konstituieren eine zwischenleibliche Lehr-LernBeziehung, die von der Trainerin gesteuert und strukturiert wird. Wie zu sehen war, erfüllt das Modaldeiktikon so in den instruierenden Handlungen der Trainerin eine aufmerksamkeitssteuernde Schlüsselfunktion. Es ist Teil einer multimodalen Konstruktion, in der es als verbaler Bestandteil auf die leiblichen Aktivitäten der Trainerin fokussiert und die visuelle Aufmerksamkeit der Schülerinnen im Vollzugsmoment einfordert. Es fungiert als flag (Streeck 2002), das die relevanten Bewegungsqualitäten und Komponenten in der Performanz der Trainerin indiziert, die die Lernenden anschließend in ihre eigene Praxis inkorporieren sollen. Da das Modaldeiktikon so ohne begleitende leibliche Handlung, auf die es referiert, unverständlich bliebe, bildet die leibliche Komponente einen unabdingbaren Bestandteil der multimodalen Konstruktion. Indem das Modaldeiktikon die visuelle Aufmerksamkeit auf die leibliche Handlung einfordert, etabliert es eine konditionelle Relevanz für den Adressatenblick, die den von Goodwin (1980, 1981) beschriebenen äußerungsinternen summons-answer-Sequenzen ähnelt (Stukenbrock 2018a, 2018b).

In den analysierten Sequenzen werden leibliche Ressourcen nicht nur als kommunikatives Ausdrucksmittel mobilisiert; sie sind zugleich Mittel und Gegenstand multimodaler Kommunikation. Eine fortwährende wechselseitige Wahrnehmung und Wahrnehmungswahrnehmung der Beteiligten sind daher zentral für die interpersonelle Koordinierung der Kommunikation und für den zwischenleiblichen Lehr-Lern-Prozess (vgl. auch Mazeland i.d.B.). Während die Wahrnehmung der Schülerinnen auf die belehrenden Demonstrationen der Trainerin gerichtet ist, überprüft die Trainerin die Aufmerksamkeitsorientierung ihrer Adressatinnen und deren Ausführung der einzuübenden Verteidigungspraktiken.

Als leibliche Aktivitäten vollziehen sich die zu erlernenden Praktiken in der Zeit und weisen eine innere Dauer auf (vgl. Streeck 2007), die Teil der zeitlichen 
Struktur der Gesamtsequenz ist. Die Analyse hat gezeigt, dass die instruierenden Handlungen der Trainerin durch Wiederholung eine Reduktion, Komprimierung und Beschleunigung erfahren (vgl. auch Deppermann i. d. B.). Dabei führen die Kondensierungs- und Beschleunigungsprozesse im ersten Paarteil zu einer Beschleunigung der Handlungen im zweiten Paarteil sowie zu einer temporalen Verdichtung und formalen Routinisierung in der sequenziellen Abfolge zwischen erstem und zweitem Paarteil, bis hin zum extrem reduzierten Drillformat. Hier sind nicht nur die jeweiligen Durchführungstempi von erstem und zweitem Paarteil erhöht, sondern auch das Abfolgetempo zwischen beiden Paarteilen.

Dabei reflektieren und konstituieren Schnelligkeit, Routinisierung und Dynamik im temporalen Verhältnis zwischen instruierender und instruierter Handlung zentrale Qualitäten erfolgreicher Selbstverteidigungspraktiken. Als Merkmale erfolgreicher Selbstverteidigung kommen sie im interaktionsgeschichtlichen Verlauf als Beschleunigungsmechanismen innerhalb der Instruktionssequenzen selbst zum Tragen. Instruierte Praktiken (Selbstverteidigungspraktiken) und Instruktionspraxis (Sequenz instruierende-instruierte-Handlung) treten in der emergierenden Interaktion in ein ikonisches, reflexives Verhältnis zueinander. Während die leiblichen Selbstverteidigungsmuster im Übungsprozess quasi automatisiert werden, werden die mobilisierten multimodalen Ausdrucksressourcen im Kommunikationsprozess abgeschliffen, reduziert und verfestigt. Dieser doppelte Sedimentierungsprozess, in dem Übungs- und Kommunikationsprozess reflexiv aufeinander bezogen sind und Erwartungserwartungen (Günthner/Knoblauch 1994: 702) zementiert werden, vollzieht sich in unterschiedlichen, gleichwohl miteinander verbundenen Domänen, der Grammatik und der Interaktion. Solche multimodalen Sedimentierungsprozesse in leiblichen Aktivitätskontexten könnten exemplarische Analysegegenstände für ein erst noch zu entwickelndes Grammatikalisierungsmodell bilden, das die Emergenz grammatischer Strukturen aus leiblichen Praktiken bzw. die Emergenz multimodaler Konstruktionen nachweist, für die das Modaldeiktikon so Pate steht.

\section{Literatur}

Auer, Peter (2005): Projection in Interaction and Projection in Grammar. In: Text, 25(1):7-36. Auer, Peter (2006): Construction Grammar meets Conversation. Einige Überlegungen am Beispiel von „so“-Konstruktionen. In: Günthner, Susanne und Wolfgang Imo (Hrsg.), Konstruktionen in der Interaktion, S. 291-314. Berlin: de Gruyter.

Auer, Peter (i. d. B.): Genau! Der auto-reflexive Dialog als Motor der Entwicklung von Diskursmarkern. In: Weidner, Beate et al. (Hrsg.), Verfestigungen in der Interaktion. Konstruktionen, sequenzielle Muster, kommunikative Gattungen. Berlin: de Gruyter. 
Bühler, Karl (1965 [1934]): Sprachtheorie. Die Darstellungsfunktion der Sprache. Stuttgart: Gustav Fischer Verlag, 2. Aufl.

Couper-Kuhlen, Elizabeth (2018): Finding a Place for Body Movement in Grammar. In: Research on Language and Social Interaction, 51(1):22-25.

Couper-Kuhlen, Elizabeth und Margret Selting (2001): Forschungsprogramm , Interaktionale Linguistik'. In: Linguistische Berichte, 187:257-87.

Couper-Kuhlen, Elizabeth und Sandra A. Thompson (i. d. B.): Ratschläge in der Alltagskommunikation: Zur Verwendung einer sedimentierten Form im Englischen. In: Weidner, Beate et al. (Hrsg.), Verfestigungen in der Interaktion. Konstruktionen, sequenzielle Muster, kommunikative Gattungen. Berlin: de Gruyter.

Deppermann, Arnulf (2006): Deontische Infinitivkonstruktionen. Syntax, Semantik, Pragmatik und interaktionale Verwendung. In: Günthner, Susanne und Wolfgang Imo (Hrsg.), Konstruktionen in der Interaktion, S. 239-262. Berlin: de Gruyter.

Deppermann, Arnulf (i. d. B.): Imperative im Deutschen: Konstruktionen, Praktiken oder social action formats? In: Weidner, Beate et al. (Hrsg.), Verfestigungen in der Interaktion. Konstruktionen, sequenzielle Muster, kommunikative Gattungen. Berlin: de Gruyter.

Deppermann, Arnulf, Reinhard Fiehler und Thomas Spranz-Fogasy (Hrsg.) (2006): Grammatik und Interaktion. Untersuchungen zum Zusammenhang von grammatischen Strukturen und Gesprächsprozessen. Radolfzell: Verlag für Gesprächsforschung.

De Stefani, Elwys und Anne-Danielle Gazin (2014): Instructional sequences in driving lessons. Mobile participants and the temporal and sequential organization of actions. In: Journal of Pragmatics, 65:63-79.

Diewald, Gabriele (1997): Grammatikalisierung. Eine Einführung in Sein und Werden grammatischer Formen. Tübingen: Niemeyer.

DWB: Deutsches Wörterbuch von Jacob und Wilhelm Grimm.

Garfinkel, Harold (2002 [1967]): Studies in Ethnomethodology. Cambridge: Polity Press.

Goffman, Erving (1963): Behavior in Public Places. Notes on the Social Organization of Gatherings. New York: The Free Press.

Goffman, Erving (1981): Forms of Talk. Philadelphia: University of Pennsylvania Press.

Goffman, Erving (1983): The interaction order. In: American Sociological Review, 48:1-17.

Goodwin, Charles (1980): Restarts, Pauses, and the Achievement of a State of Mutual Gaze at Turn-Beginning. In: Sociological Inquiry, 50(3/4):272-302.

Goodwin, Charles (1981): Conversational Organization. Interaction between Speakers and Hearers. London: Academic Press.

Goodwin, Charles (1994): Professional Vision. In: American Anthropologist, 96(3):606-633.

Goodwin, Charles (1996): Transparent Vision. In: Ochs, Elinor, Emanuel A. Schegloff und Sandra A. Thompson (Hrsg.), Interaction and Grammar, S. 370-404. Cambridge: CUP.

Goodwin, Marjorie H. (1980): Processes of mutual monitoring implicated in the production of description sequences. In: Sociological Inquiry, 50(3/4):302-217.

Goodwin, Marjorie H. (2006): Participation, affect, and trajectory in family directive/response sequences. In: Text \& Talk, 26:513-541.

Günthner, Susanne und Wolfgang Imo (Hrsg.) (2006): Konstruktionen in der Interaktion. Berlin: de Gruyter.

Günthner, Susanne und Hubert Knoblauch (1994): „Forms are the food of faith“. Gattungen als Muster kommunikativen Handelns. In: Kölner Zeitschrift für Soziologie und Sozialpsychologie, 46(4):693-723. 
Hausendorf, Heiko (2003): Deixis and speech situation revisited. The mechanism of perceived perception. In: Lenz, Friedrich (Hrsg.), Deictic Conceptualisation of Space, Time and Person, S. 249-269. Amsterdam: John Benjamins.

Hopper, Paul und Elizabeth C. Traugott (1993): Grammaticalization. Cambridge: CUP.

Keevallik, Leelo (2018): What Does Embodied Interaction Tell Us About Grammar? In: Research on Language and Social Interaction, 51(1):1-21.

Kendon, Adam (2004): Gesture. Visible Action as Utterance. Cambridge: CUP.

Kluge, Friedrich (1989): Etymologisches Wörterbuch der deutschen Sprache. Berlin: de Gruyter. Lehmann, Christian (1995): Thoughts on Grammaticalization. München: Linom Europa.

Lindwall, Oskar und Anna Ekström (2012): Instruction-in-interaction. The teaching and learning of a manual skill. In: Human Studies, 35:27-49.

Luhmann, Niklas (1984): Soziale Systeme. Frankfurt a. Main: Suhrkamp.

Meyer, Christian, Jürgen Streeck und Scott J. Jordan (Hrsg.) (2017): Intercorporeality. Emerging Socialities in Interaction. Oxford: OUP.

Mazeland, Harrie (i. d. B.): Die Aktivität als eigenständiger interaktionsorganisierender Bereich. In: Weidner, Beate et al. (Hrsg.), Verfestigungen in der Interaktion. Konstruktionen, sequenzielle Muster, kommunikative Gattungen. Berlin: de Gruyter.

Mondada, Lorenza (2009): The Embodied and Negotiated Production of Assessments in Instructed Actions. In: Research on Language and Social Interaction, 42(4):329-61.

Mondada, Lorenza (2011): The situated organization of directives in French. Imperatives and action coordination in video games. In: Nottingham French Studies, 50(2):19-50.

Ningelgen, Jana und Peter Auer (2017): Is there a multimodal construction based on non-deictic so in German? In: Linguistics Vanguard, 3(1):1-15.

Rauniomaa, Mira und Tina Keisanen (2012): Two multimodal formats for responding to requests. In: Journal of Pragmatics, 44:829-842.

Ryle, Gilbert (1949): The Concept of Mind. Chicago: The University of Chicago Press.

Ryle, Gilbert (1971 [1946]): Collected Papers (Volume 2): Kapitel Knowing How and Knowing That, S. 212-225. New York: Barnes and Nobles.

Schegloff, Emanuel (1996): Turn organization. One intersection of grammar and interaction. In: Ochs, Elinor, Emanuel A. Schegloff und Sandra A. Thompson (Hrsg.), Interaction and Grammar, S. 52-133. Cambridge: CUP.

Schönherr, Beatrix (1993): Prosodische und nonverbale Signale für Parenthesen. „Parasyntax“ in Fernsehdiskussionen. In: Deutsche Sprache, 3:223-243.

Selting, Margret et al. (2009): Gesprächsanalytisches Transkriptionssystem 2 (GAT 2). In: Gesprächsforschung - Onlinezeitschrift zur verbalen Interaktion, 10:353-402.

Selting, Margret und Elizabeth Couper-Kuhlen (2000): Argumente für die Entwicklung einer ,interaktionalen Linguistik‘. In: Gesprächsforschung - Online-Zeitschrift zur verbalen Interaktion, 1:76-95.

Streeck, Jürgen (2002): Grammars, Words, and Embodied Meanings. On the Uses and Evolution of So and Like. In: Journal of Communication, 52:581-591.

Streeck, Jürgen (2007): Geste und verstreichende Zeit. In: Hausendorf, Heiko (Hrsg.), Gespräch als Prozess. Linguistische Aspekte der Zeitlichkeit verbaler Interaktion, S. 157-180. Tübingen: Narr.

Streeck, Jürgen (2018): Grammaticalization and Bodily Action. Do They Go Together? In: Research on Language and Social Interaction, 51(1):26-32. 
Stukenbrock, Anja (2008): „Wo ist der Hauptschmerz?“ - Zeigen am eigenen Körper in der medizinischen Kommunikation. In: Gesprächsforschung - Online-Zeitschrift zur verbalen Interaktion, 9:1-33.

Stukenbrock, Anja (2009a): Referenz durch Zeigen. Zur Theorie der Deixis. In: Deutsche Sprache, 37:289-315.

Stukenbrock, Anja (2009b): Erklären - Zeigen - Demonstrieren. In: Spreckels, Janet (Hrsg.), Erklären im Kontext. Neue Perspektiven aus der Gesprächs- und Unterrichtsforschung, S. 160-176. Baltmannsweiler: Schneider-Verlag Hohengehren.

Stukenbrock, Anja (2010): Überlegungen zu einem multimodalen Verständnis der gesprochenen Sprache am Beispiel deiktischer Verwendungsweisen des Ausdrucks „so“. In: InLiSt Interaction and Linguistic Structures, 47:1-23.

Stukenbrock, Anja (2013): Sprachliche Interaktion. In: Auer, Peter (Hrsg.), Grammatik - Interaktion - Kognition, S. 217-259. Stuttgart: Metzler.

Stukenbrock, Anja (2014): Take the words out of my mouth. Verbal instructions as embodied practices. In: Journal of Pragmatics, 65:80-102.

Stukenbrock, Anja (2015): Deixis in der face-to-face-Interaktion. Berlin: de Gruyter.

Stukenbrock, Anja (2016): Deiktische Praktiken. Zwischen Interaktion und Grammatik. In: Deppermann, Arnulf, Helmuth Feilke und Angelika Linke (Hrsg.), Sprachliche und kommunikative Praktiken (Jahrbuch 2015 des Instituts für Deutsche Sprache), S. 81-126. Berlin: de Gruyter.

Stukenbrock, Anja (2018a): Forward-Looking. Where Do We Go with Multimodal Projections? In: Deppermann, Arnulf und Jürgen Streeck (Hrsg.), Modalities and Temporalities. Convergences and Divergences of Bodily Resources in Interaction, S. 31-68. Amsterdam: Benjamins.

Stukenbrock, Anja (2018b): Mobile Dual Eye-Tracking in Face-to-Face Interaction. The Case of Deixis and Joint Attention. In: Brône, Geert und Bent Oben (Hrsg.), Advances in Interaction Studies, S. 265-302. Amsterdam: John Benjamins Publishing Company.

Stukenbrock, Anja (2018c): Blickpraktiken von SprecherInnen und AdressatInnen bei der Lokaldeixis. Mobile Eye Tracking-Analysen zur Herstellung von joint attention. In: Gesprächsforschung - Online-Zeitschrift zur verbalen Interaktion, 19:132-168.

Zima, Elisabeth (2014): Gibt es multimodale Konstruktionen? Eine Studie zu [V(motion) in circles] und [all the way from X PREP Y]. In: Gesprächsforschung - Online-Zeitschrift zur verbalen Interaktion, 15:1-48.

Zima, Elisabeth und Alexander Bergs (Hrsg.) (2017): Multimodality and Construction Grammar. In: Linguistics Vanguard (Editorial of a Special Issue), 3(1). 
